\title{
Thermal and Magnetic Properties of Stress-Induced Martensites in Ni-Mn-Ga Alloys
}

\author{
V.A. Chernenko, A. Amengual*, E. Cesari*, V.V. Kokorin and I.K. Zasimchuk \\ Institute of Metal Physics, Verdnadsky str. 36, Kiev 252680, Ukraine \\ * Department de Física, Univ. de les Illes Balears, E-0707I Palma de Mallorca, Spain
}

\begin{abstract}
The stress-induced transformation of single crystals of $\mathrm{Ni}-\mathrm{Mn}-\mathrm{Ga}$ ferromagnetic Heusler ordered alloys to new martensitic structures has been investigated. The intermartensitic transformations, whenever they occur, were registered during heating by calorimetry, dilatometry and magnetic susceptibility measurement, showing up a step-like strain recovery, small endothermal effects, and an abrupt change of the low-field magnetic susceptibility. During intermartensitic transformations, between modulated and unmodulated lattices, as well as during the final transformation to the parent austenitic phase, noticeable anomalous changes in the specific magnetization were observed.
\end{abstract}

\section{INTRODUCTION}

It has been found that some ferromagnetic Heusler alloys undergo thermal and stress-induced thermoelastic martensitic transformations (MT) [1-4]. These alloys have compositions near the stoichiometric of the intermetallic $\mathrm{Ni}_{2} \mathrm{MnGa}$ compound. The structure of the induced martensite depends on the composition of the alloy and on the load: type (compression or tension) and crystallographic axis. As an example of these transformations, the parent phase of an ordered L2 $1 \mathrm{Ni}-\mathrm{Mn}$-Ga alloy transforms during cooling at $278 \mathrm{~K}$ to a tetragonal martensite with a lattice modulation of 5 layers along the [110] direction (5M-martensite [5]); increasing the compressive stress along the [110] direction, it will transform to another martensite with a 7-layer lattice modulation (7M), and afterwards into a tetragonal phase (T) (without modulation). These martensites can remain after unloading in their own temperature intervals of stability and, during heating, the reverse intermartensitic transformations take place, producing a step-wise shape recovery, before the final transformation to the parent phase $(\mathrm{P})$ is produced [3].

A variety of anomalies in the physical properties of the alloys can be detected during these processes [3,6]: For instance, the new phenomenon of lattice softening during the intermartensitic transformation from the low temperature tetragonal phase into the higher temperature martensite $7 \mathrm{M}$ was found [6]. Another interesting point is that in many Ni-Mn-Ga materials, martensitic and intermartensitic transformations occur in the ferromagnetic matrix giving rise to magnetic anomalies [4-7].

In the present work an attempt has been made to search for new types of stress-induced martensites in single crystals of $\mathrm{Ni}-\mathrm{Mn}-\mathrm{Ga}$ with special attention to their thermal and magnetic properties. Such investigations can lead to a better understanding of the relationship between structural and magnetic states of Ni-Mn-Ga shape memory materials. 


\section{EXPERIMENTAL PROCEDURE}

Three Ni-Mn-Ga alloys were prepared by induction melting under argon atmosphere and casting into a copper mold. According to chemical analysis they have the following compositions (at. \%): Ni -26.6 $\mathrm{Mn}-24.2 \mathrm{Ga}$ (alloy 1), $\mathrm{Ni}-23.5 \mathrm{Mn}-23.9 \mathrm{Ga}$ (alloy 2) and $\mathrm{Ni}-31.1 \mathrm{Mn}-17.7 \mathrm{Ga}$ (alloy 3). The single crystals were grown from the initial ingots by the Bridgman method. They were oriented in the parent phase using the Laue-method. Samples of appropriate shape, dimension and orientation were obtained by using spark-cutter and, in some cases, diamond saw. The martensitic start temperatures, $\mathrm{M}_{\mathrm{S}}$, were determined to be $173 \mathrm{~K}, 283 \mathrm{~K}$ and $446 \mathrm{~K}$ for alloy 1,2 and 3 respectively.

Low temperature $\mathrm{X}$-ray diffraction analysis of the martensitic structure of alloy 1 was performed using a diffractometer with angular position controller. In order to check the modulation type of the martensite lattice for alloys 2 and 3, electron diffraction patterns were obtained at room temperature from (100) foils. As a result, martensite in alloys 1 and 2 was found to be $5 \mathrm{M}$, and $7 \mathrm{M}$ in alloy 3 .

The compression experiments were performed using especially designed press-forms, one of them suitable for simultaneous low field magnetic susceptibility measurements. The compression axis was in all cases [110]. The cooling/heating DSC records (at $10 \mathrm{~K} / \mathrm{min}$ ) were taken from Perkin Elmer DSC-4 and DSC-7 thermal analyzers. A Perkin Elmer TMA-7 dilatometer was used for dilatometric measurements during heating after decompression. The measurement of the low field magnetic susceptibility $\chi$ (magnetic field $\mathrm{H}$ below $0.3 \mathrm{Oe}$ ) was carried out by the induction method. The specific magnetization $\sigma(T, H)$ was measured at constant temperatures by the pulse technique and at constant field $\mathrm{H}$ by the conventional Faraday type magnetic balance method.

\section{RESULTS AND DISCUSSION}

It has been recently clarified [3,5,6] that uniaxial compression at $77 \mathrm{~K}$ along the [110] axis in alloys with composition (at. \%): $\mathrm{Ni}-23.6 \mathrm{Mn}-24.9 \mathrm{Ga}$ brings the formation of tetragonal martensite $\mathrm{T}$ which, after unloading and subsequent heating transforms towards the parent phase through the following structural transformations:

$$
\mathrm{T} \stackrel{\text { at } 200 \mathrm{~K}}{\longrightarrow} 7 \mathrm{M} \stackrel{\text { at } 230 \mathrm{~K}}{\longrightarrow} 5 \mathrm{M} \stackrel{\text { at } 290 \mathrm{~K}}{\longrightarrow} \mathrm{P}
$$

Further heating of the alloy will produce the transformation of the alloy from the ferromagnetic to the paramagnetic state $\left(T_{c}=368 \mathrm{~K}\right)$ [5]. The Curie temperatures $T_{c}$ of the alloys used in this work, determined from $\chi(T)$ curves, are $368 \mathrm{~K}, 363 \mathrm{~K}$ and $356 \mathrm{~K}$, for alloys 1,2 and 3 respectively. It is worth noting that the magnetic transformation occurs in the martensitic phase in alloy 3 but in the parent phase in the other two alloys, however their Curie temperatures are very close to each other.

In Fig. 1, the measurements of magnetic susceptibility, calorimetry and dilatometry during typical heating runs are shown. They correspond to alloy 2 after compression up to $15 \mathrm{~kg} / \mathrm{mm}^{2}$ at $77 \mathrm{~K}$. These measurements show the same overall behavior observed in the alloy studied previously $[3,6]$, however there are some considerable quantitative differences (see Table 1). The martensitic tetragonal phase, $T$, can exist in a fairly wide temperature range (from Table 1 , up to $244 \mathrm{~K}$ ). Therefore, the material accumulates a large strain removed by portions during heating through the reverse intermartensitic transformation (see Fig. 1a). Such a kind of step-like shape memory effect, in which the stress-induced martensites and subsequent intermartensitic transformations induced by temperature change are involved, was firstly detected in [3] and it is confirmed by the present investigation.

In reference [5] it was shown that the martensitic transformation $5 \mathrm{M} \leftrightarrow \mathrm{P}$ is accompanied by an abrupt change of the magnetic moment of the alloy $\left(\Delta \mu \sim 0.078 \mu_{B}\right)$. So, the critical strength of the magnetic field required to induce martensite in the ferromagnetic austenite can be obtained from the value of the magnetic field shift of $M_{s}$ estimated in [5] which is equal to $\sim 0.07 \mathrm{~K} / \mathrm{kOe}$.

In this work we have measured the specific magnetization $\sigma(\mathrm{T})$ of alloy 2 during heating after unloading the alloy compressed initially at $77 \mathrm{~K}$. The results are displayed in Fig. 2 . It can be observed that in addition to an earlier anomaly on the curve caused by the transformation $5 \mathrm{M} \rightarrow \mathrm{P}$ there is also an abrupt change (about half in size of the first, near $0.04 \mu_{\mathrm{B}}$ ) when the $\mathrm{T} \rightarrow 7 \mathrm{M}$ transformation occurs. According to the Clausius-Clapeyron relationship, the values of $\Delta \mu$ and the evolved heat $\mathrm{Q}$ can be used to estimate the magnetic field shift of $A_{s}$ for the $T \rightarrow 7 \mathrm{M}$ transformation. This shift is ten times larger than that for the $5 \mathrm{M} \rightarrow \mathrm{P}$ transformation.

No anomaly on the $\sigma(\mathrm{T})$ curve was detected during the $7 \mathrm{M} \rightarrow 5 \mathrm{M}$ transformation. This means that, on the contrary to the $7 \mathrm{M} \rightarrow 5 \mathrm{M}$ transformation, the crystal lattice changes during $\mathrm{T} \rightarrow 7 \mathrm{M}$ 


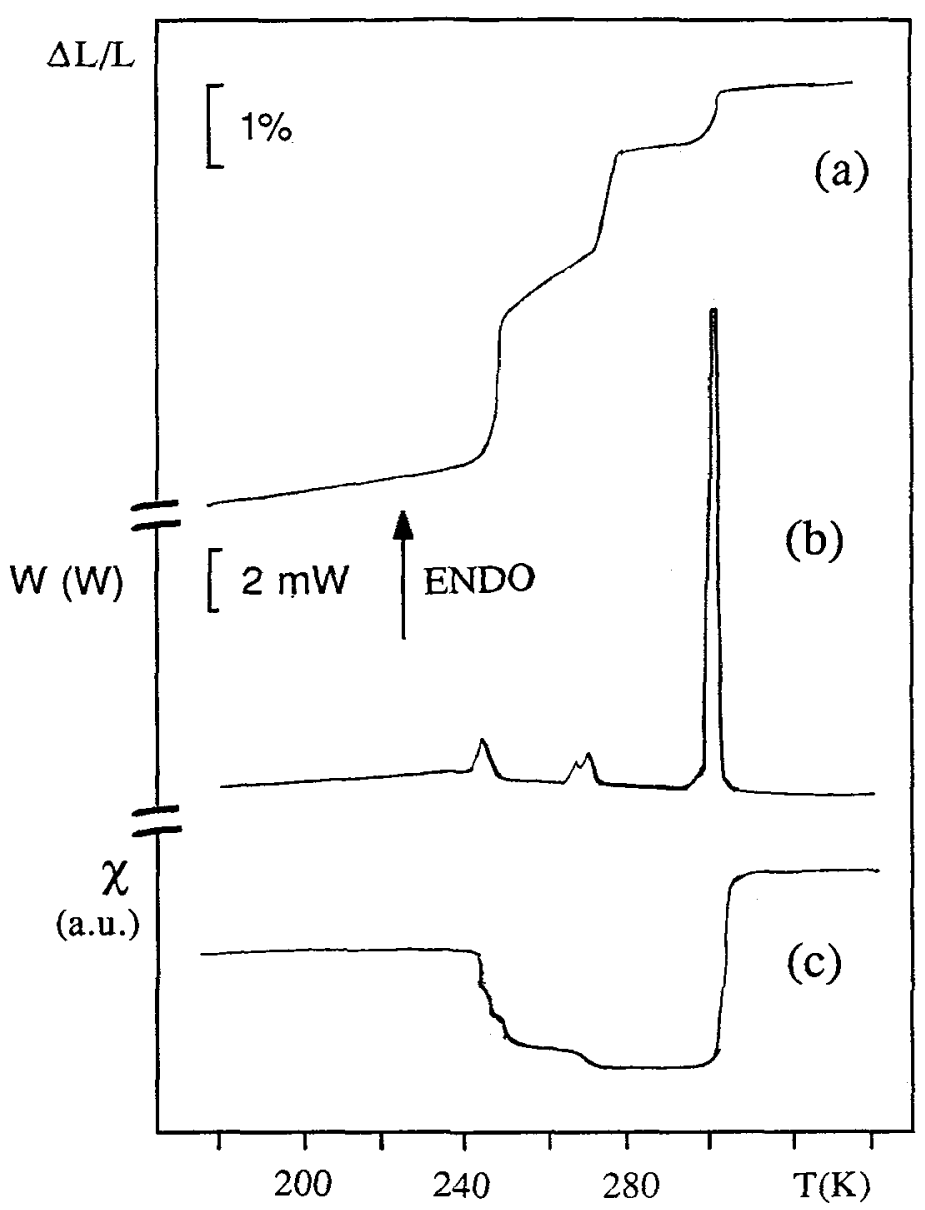

Figure 1: (a) Length change during heating of a sample from alloy 2 , initially compressed at $77 \mathrm{~K}$ along the [110] axis. (b) Heat evolved (thermogram) for the same sample during another compression and heating run. (c) Magnetic susceptibility during heating after compression.

\begin{tabular}{|c|c|c|c|c|c|c|c|}
\hline \multirow{2}{*}{ Alloy } & \multicolumn{2}{|c|}{$\mathrm{T} \rightarrow 7 \mathrm{M}$} & \multicolumn{2}{|c|}{$7 \mathrm{M} \rightarrow 5 \mathrm{M}$} & \multicolumn{2}{|c|}{$5 \mathrm{M} \rightarrow \mathrm{P}$} & $\mathrm{P} \rightarrow 5 \mathrm{M}$ \\
\hline & $A_{S}(K)$ & $\mathrm{Q}(\mathrm{J} / \mathrm{g})$ & $\mathrm{A}_{\mathrm{S}}(\mathrm{K})$ & $\mathrm{Q}(\mathrm{J} / \mathrm{g})$ & $A_{S}(K)$ & $\mathrm{Q}(\mathrm{J} / \mathrm{g})$ & $M_{S}(K)$ \\
\hline 2 & 244 & 0.22 & 270 & 0.30 & 300 & 4.2 & 283 \\
\hline Ref. $[5,6]$ & 200 & 0.37 & 230 & 0.18 & 290 & 3.8 & 278 \\
\hline
\end{tabular}

Table 1: Starting temperature and heat evolved for the martensitic and intermartensitic transformations in two Ni-Mn-Ga alloys. (Composition of sample of Ref. [5,6] (at. \%): Ni-23.6 Mn-24.9 Ga.) 
Figure 2: Specific magnetization as a function of the temperature for $\mathrm{Ni}-\mathrm{Mn}-\mathrm{Ga}$ alloy 2 during heating: without preliminary compression (curve 1); and after preliminary compression at $77 \mathrm{~K}$ with stress level $\sim 15 \mathrm{~kg} / \mathrm{mm}^{2}$ along [110] axis (curve 2).

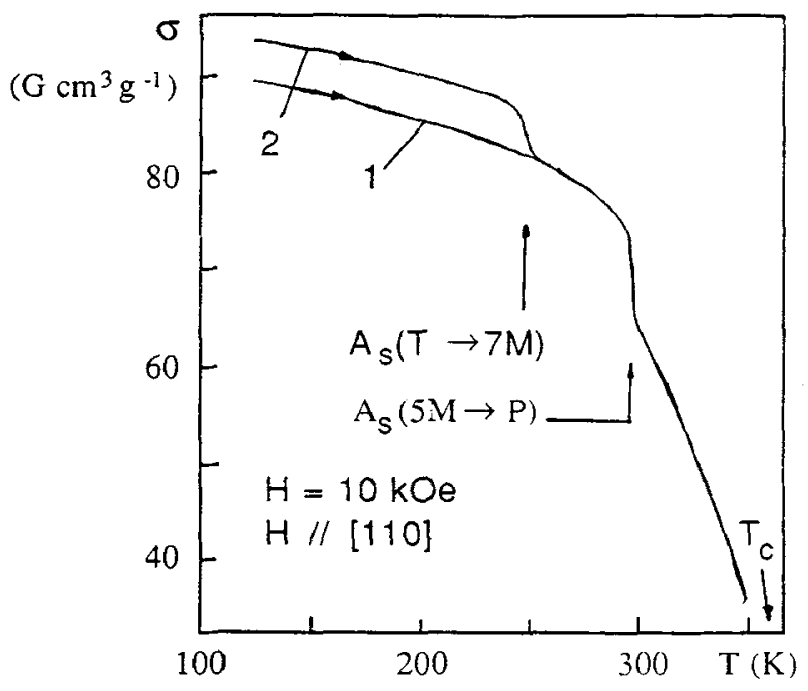

and $5 \mathrm{M} \rightarrow \mathrm{P}$ transformations produce noticeable alterations in the magnetic state of the alloy as can be seen in Figs. 1c and 2. It is worth noting again how close are the Curie temperatures for the $7 \mathrm{M}$ martensite in alloy 3 and for the parent phase in alloys 1 and 2 . The same level of magnetic interactions in the above mentioned phases can be responsible for that.

New stress-induced martensites where not observed during compression at $77 \mathrm{~K}$ and $300 \mathrm{~K}$ along the [110] axis of alloys 1 and 3. There are some experimental problems involved which need further attention: In the case of alloy 1 , the maximum applied load $\left(-20 \mathrm{Kg} / \mathrm{mm}^{2}\right)$ perhaps was not large enough. In addition, many difficulties arise in producing a perfect single crystal of alloy 3 , and we have some doubts about the alloy used in this work, its crystallographic axis could not have been the same throughout the sample. The possibility of new stress-induced martensites in these alloys cannot be yet ruled out, and further studies are in order.

\section{Conclusion}

Several martensitic phases in ferromagnetic Ni-Mn-Ga alloys were induced by combining uniaxial compression along the [110] axis and temperature. The intermartensitic transformations are accompanied by anomalies on the thermal and magnetic properties of the alloy. During the intermartensitic transformation $T \rightarrow 7 \mathrm{M}$ and the reverse transformation to the parent phase $(5 \mathrm{M} \rightarrow \mathrm{P})$ a considerable change in the magnetic properties of the alloy has been recorded.

\section{Acknowledgements}

The authors are thankful to Val'kov V.I. for his kind assistance in doing magnetization measurements. Chernenko V.A. is grateful to the Univ. Illes Balears for financing his stay at the Dept. de Fisica, UIB.

\section{References}

[1] Webster P. J. Ziebeck K. R. A., Town S. L. and Peak M. S., Phil. Mag. B49 (1984),295-310.

[2] Zasimchuk I. K., Kokorin V. V., Martynov V. V., Tkachenko A. V. and Chernenko V. A., Fiz Met. $i$ Metalloved. 6 (1990) 110-114.

[3] Kokorin V. V., Martynov V. V. and Chernenko V. A., Scr. Met. Mat. 26 (1992) 175-177.

[4] Kokorin V. V. and Chernenko V. A., Phys. Met. Metall. 68 (1989) 111-115.

[5] Chernenko V. A. and Kokorin V. V., "Ni2 $\mathrm{MnGa}$ as a new ferromagnetic ordered shape memory alloy", International Conference on Martensitic Transformations, Monterey CA 20-24 July 1992 (Monterey Inst. for Advanced Studies, 1994) pp. 1205-1210.

[6] Vasil'ev A. N., Kayper A., Kokorin V. V., Chernenko V. A., Takagi T. and Tani J., JETP Letters 58 (1993) 306-309.

[7] Vasil'ev A. N., Kokorin V. V., Savchenko Yu. I. and Chernenko V. A., Sov. Phys. JETP 71 (1990) 803-805.. 